\section{Prematuridade como fator de risco para pressão arterial elevada em crianças: uma revisão sistemática}

\author{
Preterm birth as a risk factor for high blood \\ pressure in children: a systematic review
}

\author{
1 Programa de Pós-graduação \\ em Saúde Coletiva, \\ Universidade Federal do \\ Espírito Santo, Vitória, Brasil. \\ 2 Programa de Pós-graduação \\ em Ciências Fisiológicas, \\ Universidade Federal do \\ Espírito Santo, Vitória, Brasil. \\ Correspondência \\ M. C. B. Molina \\ Programa de Pós-graduação \\ em Saúde Coletiva, \\ Universidade Federal do \\ Espírito Santo. \\ Av. Marechal Campos 1468 , \\ Vitória, ES 29040-090, Brasil. \\ mdmolina@uol.com.br
}

\begin{abstract}
Epidemiological studies have suggested that arterial hypertension is a chronic disease that begins in childhood, and that prematurity (birth at less than 37 weeks' gestational age) is potentially associated with the development of hypertension in childhood and adulthood. Our objective was to identify the association between prematurity and high blood pressure in children, using a systematic literature review. Original articles related to the theme and published in English, Portuguese, or Spanish from 1998 to 2009 were selected from the MEDLINE, LILACS, and SciELO databases. We excluded articles without abstracts, review articles, and articles not related to prematurity and hypertension in childhood. Nine articles were located and analyzed: 5 case-control studies, 2 cross-sectional studies, and 2 cohort studies. The majority of the studies failed to show an association between prematurity and arterial hypertension in childhood. However, the influence of prematurity should not be ruled out, given the small number of studies on this theme and the diversity of methodological approaches in the literature.
\end{abstract}

Hypertension; Premature Infant; Child

\author{
Anna Paula Coelli 1 \\ Larissa Rangel Nascimento 1 \\ Jose Geraldo Mill 2 \\ Maria del Carmen Bisi Molina 1
}

\section{Introdução}

Estudos epidemiológicos têm sugerido que a hipertensão arterial do adulto tem início na infância 1,2 . Porém, diferentemente do que ocorre em adultos, nos quais os fatores determinantes da hipertensão arterial são razoavelmente bem conhecidos, com parâmetros de normalidade estabelecidos internacionalmente, ainda há muitas controvérsias em relação à prevalência, à magnitude e aos fatores de riscos associados a este agravo em crianças 3 .

Há poucos anos, a hipertensão arterial na infância era tratada como um evento raro e, na maioria das vezes, secundário a afecções renais, cardíacas ou endócrinas. Essa concepção tem sido questionada em diferentes estudos que têm evidenciado aumento na prevalência de hipertensão arterial essencial na faixa etária pediátrica 4,5,6, ainda que utilizando diferentes parâmetros para o seu diagnóstico.

Nas últimas duas décadas, alguns estudos evidenciaram associação entre prematuridade, condição de crianças nascidas com idade gestacional inferior a 37 semanas 7 , e o desenvolvimento de alguns agravos, incluindo hipertensão arterial, intolerância à glicose e dislipidemias, tanto em crianças 8,9,10 como em adultos 11,12,13.

Tal questão merece destaque, tendo em vista que no mundo nascem diariamente aproximadamente 13 milhões de prematuros $(10 \%$ dos nascimentos), de acordo com a revisão so- 
bre o assunto publicada no Bulletin of the World Health Organization em 2010 14. No sul da África essa taxa atinge $17,5 \%$, nos Estados Unidos, $10,6 \%$ e na Europa, $6,2 \% 14$. A prematuridade contribui, ainda, com $28 \%$ da taxa de mortalidade em crianças menores de cinco anos, sendo a principal causa de morte nesta faixa etária 7 . De acordo com o Ministério da Saúde, a proporção de nascidos vivos prematuros permanece estável no Brasil desde 2000, apresentando uma taxa média de $6,6 \% 15$.

O desenvolvimento de terapias intensivas no cuidado neonatal nas últimas décadas elevou a taxa de sobrevida de prematuros 13. Apesar do avanço da tecnologia voltada para essa área da medicina, os efeitos da prematuridade ao longo da vida são ainda pouco conhecidos. Mais escasso ainda é o conhecimento dos efeitos da prematuridade sobre as doenças crônicas em fases posteriores da vida. Poucos estudos têm abordado a contribuição do tempo de gestação na determinação do risco à saúde na vida adulta 16, principalmente pela necessidade de se acompanhar, por um longo período de tempo, o grupo de indivíduos.

Ainda que de difícil condução, alguns desses estudos têm evidenciado a associação entre a prematuridade e o aumento do risco para as doenças cardiovasculares e outros agravos crônicos. Johansson et al. ${ }^{12}$, estudando uma grande amostra de homens na Suécia, identificaram que o nascimento prematuro constitui fator de risco para hipertensão arterial e este risco era particularmente elevado nos homens que nasceram com, pelo menos, oito semanas antes do tempo esperado. Homens nascidos antes de 29 semanas de gestação tiveram risco duas vezes maior de apresentar pressão arterial sistólica elevada (PAS $\geq 140 / 90 \mathrm{mmHg}$ ). Por sua vez, os nascidos entre 29 e 32 semanas e aqueles entre a 33a e a $36 \underline{a}$ semanas de gestação apresentaram, respectivamente, $48 \%$ e $24 \%$ mais chances de apresentarem PAS elevada. A associação entre idade gestacional e PAS prevaleceu mesmo depois de controlado o fator familiar como idade da mãe ao nascimento, níveis educacional e socioeconômico familiar 12 .

Outro estudo que evidenciou essa associação foi o de Irving et al. 11. Foram observados maiores valores de PAS e de glicemia em jejum em crianças prematuras em relação àquelas que nasceram a termo. Delziel et al. 13 também encontraram associação estatisticamente significante entre prematuridade e PAS, mesmo após ajuste por sexo e peso ao nascer, em estudo de coorte com adultos aos 30 anos de idade. Em outro estudo 17, essa associação manteve-se mesmo após ajuste para fatores genéticos (irmãos e não-irmãos), posição socioeconômica, fatores da vida materna e relacionados à gestação (idade materna ao nascimento, número de gestações e complicações durante a gestação).

Um padrão de crescimento vascular anormal 18, subdesenvolvimento de reflexos autonômicos 19 e anormalidades renais 20 nos prematuros estão sendo estudados como possíveis causas da elevação dos níveis pressóricos em fases posteriores da vida. Além disso, a hiperatividade da função simpático-adrenal, devido ao estresse sofrido no período perinatal e nas Unidades de Terapias Intensivas, também pode ser considerada como fator predisponente ao surgimento da hipertensão arterial 21 .

Outros fatores na vida pré-natal, como o baixo peso ao nascer, também determinariam alterações metabólicas e/ou renais persistentes ao longo da vida 22,23. Nesse sentido, Barker et al. 24 sugerem que estímulos aplicados em períodos críticos do desenvolvimento humano, como a gestação, resultariam em efeitos duradouros sobre a estrutura e função de órgãos e sistemas. Como existe relação entre peso ao nascimento e idade gestacional, os efeitos e conseqüências destas variáveis na vida futura podem ser de difícil distinção 12,18.

A relação entre o baixo peso ao nascer e o desenvolvimento de hipertensão arterial essencial em fases posteriores da vida tem sido mais estudada do que a relação entre idade gestacional e hipertensão arterial 16. Johansson et al. 12 argumentam, porém, que a elevada mortalidade em prematuros e deficiências metodológicas, como a falta de ajuste para o peso atual, podem ter levado à superestimação da associação entre peso ao nascer e alterações da pressão arterial ao longo vida. $\mathrm{O}$ fato é que crianças prematuras estão mais sujeitas aos agravos futuros advindos da própria condição da prematuridade, como conseqüência da imaturidade de órgãos e danos advindos do baixo peso ao nascer associado.

Levando em consideração resultados de pesquisas realizadas com crianças e de evidências da elevação da pressão arterial nesse grupo, bem como da possível associação da hipertensão com prematuridade, o presente estudo teve como objetivo realizar uma revisão sistemática sobre esta temática.

\section{Metodologia}

\section{Seleção de artigos}

Foram selecionados, por meio de busca eletrônica, artigos das bases de dados MEDLINE, LILACS e SciELO publicados de 1998 a 2009. Para o levantamento foram utilizados os seguintes descri- 
tores: premature birth and hypertension; premature birth and blood pressure; gestacional age and blood pressure; preterm birth and blood pressure. Os critérios de exclusão para a seleção dos artigos foram: (1) artigos que não apresentavam resumo, (2) artigos de revisão, e (3) artigos indisponíveis em português, inglês ou espanhol. Para serem incluídos na análise, os trabalhos deveriam ter sido planejados para avaliar a associação entre prematuridade e elevação da pressão arterial.

Os termos descritores foram utilizados separadamente no campo de busca de cada base de dados, sendo utilizadas as ferramentas de refinamento quando estas estavam disponíveis, como nas bases MEDLINE e LILACS. Foram selecionados apenas os estudos desenvolvidos com população infantil. Todavia, a amplitude das faixas etárias incluiu idades que variaram de 2 a 17 anos, ou seja, crianças e adolescentes 25 . Na base de dados SciELO, como não há a opção de limitar a busca dos estudos para crianças, os artigos foram limitados somente pelo ano de publicação e selecionados posteriormente de acordo com os temas e população estudada. Dos 158 artigos encontrados na primeira fase de seleção, 107 foram identificados após aplicação dos critérios de exclusão.

Utilizando o descritor premature birth and hypertension foram encontrados 15 artigos no MEDLINE, tendo sido excluídos 5 por serem revisões e outro por não apresentar resumo, e 2 artigos na base de dados LILACS. Utilizando o mesmo termo descritor, foram encontrados 2 artigos nas bases do SciELO. Em busca posterior, usamos o termo premature birth and blood pressure, sendo encontrados 18 artigos no MEDLINE, dos quais, após utilização dos mesmos critérios de seleção, permaneceram 15 para consulta. Foram encontrados 8 artigos na SciELO, dos quais 2 eram repetidos, e nenhum artigo na LILACS.

Com o descritor gestacional age and blood pressure foram encontrados 62 artigos na base de dados MEDLINE, sendo excluídos 3 por não apresentarem resumo, 8 por serem repetidos, 1 artigo por ser de revisão e 1 por estar escrito em húngaro. Foram encontrados 27 artigos na base de dados SciELO e 1 artigo na base de dados LILACS. Usando-se preterm birth and blood pressure como descritores, foram encontrados 16 artigos na MEDLINE, sendo 10 destes retirados da análise por serem repetidos; 7 na SciELO, dos quais 2 eram repetidos e nenhum na LILACS.

Após a leitura dos resumos dos 122 artigos selecionados, foram excluídos 108 por não se adequarem ao tema estudado. Assim, foram excluídos os artigos que não apresentassem em sua análise nenhuma proposta de associar prematuridade com elevação da pressão arterial em crianças e/ou adolescentes. Ao final da busca foram selecionados para esta análise 14 estudos, sendo 9 de caso-controle, 2 estudos transversais e 3 estudos de coorte prospectiva. Após a leitura dos 14 artigos foram excluídos da análise 5. Os estudos de Rakow et al. 10, Bonamy et al. 26, Fattal-Valesvki et al. 27, De Rogalski et al. 28 e Bergel et al. 29 foram retirados da análise por não possuírem como objetivo a associação da pressão arterial com a idade gestacional.

\section{Resultados}

A Tabela 1 mostra as características gerais dos 9 estudos utilizados na presente análise, incluindo tipo de estudo, ano de publicação, método de estimativa da idade gestacional, critério para a classificação de prematuridade e método de aferição da pressão arterial. Com exceção da pesquisa de Rondó et al. ${ }^{30}$, todos os estudos foram realizados no exterior, sendo que 5 foram conduzidos apenas com crianças e 4 com crianças e adolescentes. A maneira como foram feitas as estimativas da idade gestacional variou entre os estudos, sendo identificados quatro métodos: ultrassonografia (2 estudos); ultrassonografia e data da última menstruação (2 estudos); somente pela data da última menstruação (1 estudo); por registros (2 estudos); e por dado coletado em entrevista (1 estudo). Um dos artigos não mencionou qual recurso foi utilizado para o cálculo da idade gestacional 31 .

Quanto à aferição da pressão arterial, em 8 dos 9 estudos foi utilizado dispositivo oscilométrico, sendo que 2 trabalhos 9,16 utilizaram a Monitorização Ambulatorial da Pressão Arterial (MAPA). Bracewell et al. 31 usaram o método auscultatório. Quanto ao número de aferições da pressão arterial, foram encontrados os seguintes números de medidas: um realizou apenas uma medida da pressão arterial, dois utilizaram a média de duas aferições, um fez três aferições, um fez seis aferições e em um foram realizadas sete medidas. No estudo de Bracewell et al. 31 não foi mencionado o número de medidas realizadas. Em relação à posição adotada durante as aferições, a maioria optou pela posição supina. Dentre os estudos que realizaram a medida clínica da pressão arterial, o tempo de repouso antes da realização da medida para pressão arterial, descrito na Tabela 1 , foi mencionado por Cheung et al. 8, Johansson et al. 21 e Rondó et al. 30.

Pode-se observar na Tabela 2 que somente 2 estudos associaram estatisticamente prematuridade e pressão arterial. Relton et al. 16 encontraram valores elevados de PAS em crianças prematuras após ajuste para peso ao nascer, com 
Tabela 1

Características dos artigos selecionados para a revisão.

\begin{tabular}{|c|c|c|c|c|c|c|}
\hline $\begin{array}{l}\text { Estudos de } \\
\text { caso-controle }\end{array}$ & Local (ano) & Amostra & Pareamento & $\begin{array}{c}\text { Estimativa da } \\
\text { idade gestacional }\end{array}$ & Prematuridade & $\begin{array}{c}\text { Aferição da } \\
\text { pressão arterial }\end{array}$ \\
\hline Bayrakci et al. 9 & Turquia (2007) & $\mathrm{n}=41 ; 5-17$ anos & $\begin{array}{c}\text { Pareados por } \\
\text { peso, altura e } \\
\text { antecedentes } \\
\text { socioeconômicos; } \\
\text { Caso: crianças } \\
\text { prematuras com } \\
\text { ou sem RCIU; } \\
\text { Controle: crianças } \\
\text { a termo e com } \\
\text { PAIG }\end{array}$ & Ultrassonografia & $<37$ semanas & $\begin{array}{c}\text { MAPA; HA > p95. } \\
\text { As medições } \\
\text { foram realizadas a } \\
\text { cada } 20 \text { minutos } \\
\text { durante o dia } \\
\text { (08:00-20:00) e a } \\
\text { cada } 30 \text { minutos } \\
\text { durante a noite } \\
\text { (24:00-06:00) }\end{array}$ \\
\hline Cheung et al. 8 & Hong Kong (2004) & $\begin{array}{c}\mathrm{n}=86 ; 5-12 \text { anos } \\
\quad(\text { média }=8,2)\end{array}$ & $\begin{array}{l}\text { 1) prematuros } \\
\text { e PIG; 2) } \\
\text { prematuros com } \\
\text { PAIG; 3) a termo } \\
\text { com PAIG }\end{array}$ & $\begin{array}{c}\text { Data do último } \\
\text { período menstrual }\end{array}$ & $\begin{array}{c}\text { Grupo 1: } 32 \\
\text { semanas; Grupo } \\
\text { 2: } 29,4 \text { semanas }\end{array}$ & $\begin{array}{c}\text { Dispositivo } \\
\text { oscilométrico. } \\
\text { Feitas } 2 \text { aferições } \\
\text { com a criança } \\
\text { deitada, na } \\
\text { mesma ocasião. } \\
\text { Média de } 2 \\
\text { leituras. Repouso } \\
\text { de } 15 \text { minutos } \\
\text { para as medidas }\end{array}$ \\
\hline Johansson et al. 21 & Suécia (2007) & $\begin{array}{c}\mathrm{n}=105 \text { crianças; } \\
\text { 9-10 anos (média } \\
\text { de idade }=9,6 \\
\text { anos) }\end{array}$ & $\begin{array}{c}\text { Pareados por } \\
\text { idade, nascimento } \\
\text { e sexo. 1) a } \\
\text { termo com PAIG } \\
\text { (controle); 2) a } \\
\text { termo e PIG } \\
3 \text { prematuros }\end{array}$ & Ultrassonografia & $\mathrm{IG}<30$ semanas & $\begin{array}{c}\text { Dispositivo } \\
\text { oscilométrico. } \\
\text { Feitas } 3 \text { aferições } \\
\text { com a criança em } \\
\text { jejum e deitada, } \\
\text { repouso de } 15 \\
\text { minutos antes da } \\
\text { aferição. Média } \\
\text { das } 3 \text { leituras. } \\
\text { Três aferições } \\
\text { consecutivas com } \\
\text { a criança em pé } \\
\text { e uma aferição } \\
\text { realizada após o } \\
\text { teste de estresse } \\
\text { mental }\end{array}$ \\
\hline Bracewell et al. 31 & $\begin{array}{l}\text { Reino Unido e } \\
\text { Irlanda (2007) }\end{array}$ & $\begin{array}{c}n=241 \text { casos e } \\
160 \text { controles; } \\
6 \text { anos }\end{array}$ & $\begin{array}{c}\text { Pareados por } \\
\text { idade e sexo. } \\
\text { Caso }=241 \\
\text { prematuros com } \\
\leq 25 \text { semanas de } \\
\text { IG; Controle }= \\
160 \text { nascidos a } \\
\text { termo }\end{array}$ & & $\leq 25$ semanas & $\begin{array}{c}\text { Aferida com } \\
\text { as crianças } \\
\text { sentadas com } \\
\text { esfigmomanô- } \\
\text { metro de mercúrio }\end{array}$ \\
\hline
\end{tabular}

(continua) 
Tabela 1 (continuação)

\begin{tabular}{|c|c|c|c|c|c|c|}
\hline $\begin{array}{l}\text { Estudos de } \\
\text { caso-controle }\end{array}$ & Local (ano) & Amostra & Pareamento & $\begin{array}{c}\text { Estimativa da } \\
\text { idade gestacional }\end{array}$ & Prematuridade & $\begin{array}{c}\text { Aferição da } \\
\text { pressão arterial }\end{array}$ \\
\hline Bonamy et al. 34 & Suécia (2007) & $\mathrm{n}=60 ; 7-12$ anos & $\begin{array}{l}\text { Pareados por ano } \\
\text { de nascimento e } \\
\text { sexo. Casos = } 39 \\
\text { muito prematuros; } \\
\text { Controles = } 21 \mathrm{a} \\
\text { termos }\end{array}$ & Registros & $\begin{array}{l}29 \text { semanas } \\
\text { (média) }\end{array}$ & $\begin{array}{l}\text { Uma média de } \\
\text { seis medidas } \\
\text { consecutivas no } \\
\text { braço esquerdo, } \\
\text { tomadas a } \\
\text { intervalos de } \\
3 \text { minutos, foi } \\
\text { considerada } \\
\text { como PA do } \\
\text { sujeito. Utilizado } \\
\text { o método } \\
\text { oscilométrico } \\
\text { automático. } \\
\text { Posição semi- } \\
\text { supina }\end{array}$ \\
\hline Estudos seccionais & Local (ano) & Amostra & $\begin{array}{c}\text { Forma de coleta } \\
\text { de dados }\end{array}$ & $\begin{array}{c}\text { Estimativa da } \\
\text { idade gestacional }\end{array}$ & Prematuridade & $\begin{array}{l}\text { Aferição da } \\
\text { pressão arterial }\end{array}$ \\
\hline Relton et al. 16 & $\begin{array}{l}\text { Reino Unido } \\
\text { (2008) }\end{array}$ & $\begin{array}{c}\mathrm{n}=483 ; 6-16 \\
\text { anos (media }= \\
11,2 \text { anos) }\end{array}$ & $\begin{array}{c}\text { Questionário } \\
\text { aplicado aos pais }\end{array}$ & $\begin{array}{l}\text { Estimado pela } \\
\text { data da última } \\
\text { menstruação } \\
\text { e por } \\
\text { ultrassonografia }\end{array}$ & $<37$ semanas & $\begin{array}{l}\text { MAPA. Medidas } \\
\text { realizadas a } \\
\text { cada } 30 \text { minutos } \\
\text { durante o dia e } 60 \\
\text { minutos durante } \\
\text { a noite }\end{array}$ \\
\hline Willemsen et al. 32 & $\begin{array}{c}\text { Países Baixos } \\
\text { (2008) }\end{array}$ & $\mathrm{n}=479 ; 4-9$ anos & $\begin{array}{c}\text { Coleta de } \\
\text { dados primários } \\
\text { (antropometria } \\
\text { e medições da } \\
\text { pressão arterial, e } \\
\text { dados bioquímicos) }\end{array}$ & $\begin{array}{l}\text { Determinada por } \\
\text { ultrassonografia } \\
\text { no primeiro } \\
\text { trimestre, se } \\
\text { disponível, ou } \\
\text { outro cálculo } \\
\text { a partir da } \\
\text { data da última } \\
\text { menstruação }\end{array}$ & $<36$ semanas & $\begin{array}{l}\text { Dispositivo } \\
\text { oscilométrico. } \\
\text { As PA sistólica e } \\
\text { diastólica foram } \\
\text { medidas duas } \\
\text { vezes no braço } \\
\text { esquerdo. A } \\
\text { média das duas } \\
\text { medições foi } \\
\text { utilizada para } \\
\text { análise }\end{array}$ \\
\hline Estudos de coorte & Local (ano) & Amostra & $\begin{array}{c}\text { Tempo de } \\
\text { acompanhamento }\end{array}$ & $\begin{array}{c}\text { Forma de coleta } \\
\text { de dados }\end{array}$ & Prematuridade & $\begin{array}{c}\text { Aferição da } \\
\text { pressão arterial }\end{array}$ \\
\hline Woelk et al. 33 & Zimbábue (1998) & $\begin{array}{c}\mathrm{n}=756 ; \text { crianças } \\
\text { com média de } 6,5 \\
\text { anos }\end{array}$ & & Registros & $<37$ semanas & $\begin{array}{c}\text { Dispositivo } \\
\text { oscilométrico. } \\
\text { Três aferições com } \\
\text { as crianças em } \\
\text { posição supina, } \\
\text { média das duas } \\
\text { últimas medidas. } \\
\text { Intervalo de } 2 \\
\text { minutos entre as } \\
\text { medidas }\end{array}$ \\
\hline
\end{tabular}

(continua) 
Tabela 1 (continuação)

\begin{tabular}{|c|c|c|c|c|c|c|}
\hline Estudos de coorte & Local (ano) & Amostra & $\begin{array}{c}\text { Tempo de } \\
\text { acompanhamento }\end{array}$ & $\begin{array}{l}\text { Forma de coleta } \\
\text { de dados }\end{array}$ & Prematuridade & $\begin{array}{c}\text { Aferição da } \\
\text { pressão arterial }\end{array}$ \\
\hline Rondó et al. 30 & Brasil (2008) & $n=472 ; 5-8$ anos & 23 meses & Entrevistas & $<37$ semanas & $\begin{array}{c}\text { O exame } \\
\text { foi realizado } \\
\text { com a criança } \\
\text { deitada após } \\
\text { repouso de } 10 \\
\text { minutos. Método } \\
\text { oscilométrico } \\
\text { (HDI/Pulse } \\
\text { Wave CR-2000). } \\
\text { Classificação da } \\
\text { PA pelo NHBPEP }\end{array}$ \\
\hline
\end{tabular}

HA: hipertensão arterial; IG: idade gestacional; MAPA: Monitorização Ambulatorial da Pressão Arterial; NHBPEP: National High Blood Pressure Education

Program; PA: pressão arterial; PAIG: peso adequado para a idade gestacional; PIG: pequeno para a idade gestacional; RCIU: restrição de crescimento

intra-uterino.

um aumento de $6,2 \mathrm{mmHg}$ para cada semana de prematuridade na gestação. Essa associação permaneceu significativa após ajuste por idade e índice de massa corporal (IMC), ainda que a idade gestacional não tenha sido associada significativamente ao aumento da pressão arterial diastólica (PAD). Willemsen et al. 32 observaram que prematuros e pequenos para idade gestacional (PIG) apresentaram valores significativamente maiores de PAS e PAD do que crianças nascidas PIG, porém nascidas a termo. A significância permaneceu após ajuste para possíveis fatores de confusão (idade, sexo, etnia, peso ao nascer, comprimento ao nascer, IMC e estatura atuais). Além disso, o porcentual das crianças com pressão arterial sistólica elevada foi maior nos prétermos e nos PIG (26,3\%) em comparação com as crianças a termo e PIG (16,8\%). As análises de regressão múltipla indicaram que a idade gestacional contribuiu significativamente para a variância das PAS e PAD.

Bayrakci et al. 9, Johansson et al. 21 e Woelk et al. 33 não encontraram associação estatisticamente significante entre idade gestacional e pressão arterial. Cheung et al. 8, por sua vez, encontraram valores mais altos de pressão arterial somente no grupo de prematuros PIG; o grupo de prematuros com peso adequado ao nascimento não diferiu do grupo de crianças a termo (dados não apresentados), sugerindo que crianças, cujo peso ao nascimento é adequado para a gestação, não apresentaram maior chance para o desenvolvimento de hipertensão arterial. Bracewell et al. 31, apesar de terem encontrado uma maior variabilidade de PAS em crianças prematuras (dados não apresentados), não observaram diferenças significativas nos valores de PAS e PAD aos seis anos de idade entre os nascidos prematuros e aqueles nascidos a termo.

Em análise de regressão múltipla, Bonamy et al. 34 identificaram a existência de associação significativa somente entre PAS e prematuridade. Porém, ao ajustar o modelo pelo IMC, a associação não se manteve significante. Rondó et al. 30 observaram correlação fraca entre PAS e PAD e idade gestacional.

Os artigos utilizados nessa análise estão listados na Tabela 3 de acordo com os fatores de confundimento utilizados nas análises estatísticas de regressão. Dois estudos, Bayrakci et al. ${ }^{9}$ e Rondó et al. ${ }^{30}$, utilizaram somente análise univariada. Dentre os fatores de confundimento analisados, os mais citados foram altura atual (4 artigos), seguidos do IMC (3 artigos), e sexo e idade, ambos citados por 2 artigos.

\section{Discussão}

A busca por artigos para realizar esta revisão demonstrou a escassez de estudos que objetivaram analisar a relação entre prematuridade e elevação da pressão arterial em crianças. A maior parte dos artigos selecionados (7 dos 9) foi publicada em 2007 ou 2008, dado que demonstra ser esse um tema de interesse bastante recente. A escassez de trabalhos, bem como a atualidade do tema, podem estar relacionadas ao aumento da sobrevida de prematuros propiciado pelo avanço de tecnologias na área da saúde nas últimas décadas. Soma-se a essa questão, o fato de que a hipertensão arterial essencial na infância começou a receber a devida atenção da pediatria somente nos últimos 25 anos 4,5,6,13. 
Associação entre prematuridade e elevação da pressão arterial nos artigos selecionados.

\begin{tabular}{|c|c|c|c|c|}
\hline Artigo & Desfecho & Variáveis de interesse & Análise estatística & Valor de $p$ \\
\hline Bayrakci et al. 9 & Pressão arterial & Prematuridade & $\begin{array}{l}\text { PA 24h; teste t de Student; } \\
\text { análise univariada }\end{array}$ & $>0,05$ \\
\hline \multirow[t]{2}{*}{ Cheung et al. 8} & Pressão arterial & Prematuridade; prematuros e PIG & Análise de variância & $<0,001$ \\
\hline & & & Ajuste por idade e VOP & 0,029 \\
\hline Johansson et al. 12 & Pressão arterial & Prematuridade & $\begin{array}{l}\text { Análise de variância e teste t } \\
\text { de Student Ajuste para altura } \\
\text { atual e eclâmpsia }\end{array}$ & $>0,05$ \\
\hline \multirow[t]{4}{*}{ Relton et al. 16} & Pressão arterial & Prematuridade & $\begin{array}{l}\text { Regressão linear ajustada por } \\
\text { semana de gestação }\end{array}$ & 0,063 \\
\hline & & & PAS não ajustada & 0,036 \\
\hline & & & $\begin{array}{l}\text { Ajuste para peso ao nascer, } \\
\text { idade atual e IMC }\end{array}$ & $<0,05$ \\
\hline & & & PAD & 0,356 \\
\hline \multirow[t]{5}{*}{ Bracewell et al. 31} & Pressão arterial & Prematuridade & Regressão residual & \\
\hline & & & PAS não ajustada & 0,043 \\
\hline & & & Ajustada por altura e IMC & $>0,05$ \\
\hline & & & PAD não ajustada & 0,015 \\
\hline & & & Ajustada por altura e IMC & $>0,05$ \\
\hline \multirow[t]{2}{*}{ Woelk et al. 33} & Pressão arterial & Prematuridade & $\begin{array}{l}\text { Variância (regressão linear } \\
\text { múltipla; ajustada para peso } \\
\text { atual) }\end{array}$ & $>0,05$ \\
\hline & & & $\begin{array}{l}\text { Idade gestacional divida em } \\
\text { tercis ( }<38 \text { semanas, } \geq 38 \text { e } \\
\geq 39 \text { semanas, }>40 \text { e } \leq 44 \\
\text { semanas, }<37 \text { semanas) }\end{array}$ & $>0,05$ \\
\hline Rondó et al. 30 & Pressão arterial & Prematuridade & Correlação (PAS, PAD) & $>0,05$ \\
\hline \multirow[t]{10}{*}{ Bonamy et al. 26} & Hipertensão arterial & Prematuridade & $\begin{array}{l}\text { Qui-quadrado, t-Student, } \\
\text { variância }\end{array}$ & \\
\hline & & & PAS & 0,33 \\
\hline & & & PAD & 0,6 \\
\hline & & & Regressão múltipla & \\
\hline & & & $\begin{array}{l}\text { Ajustada para sexo, idade, } \\
\text { altura, história familiar } \\
\text { para as DCV }\end{array}$ & \\
\hline & & & PAS & 0,010 \\
\hline & & & PAD & $>0,05$ \\
\hline & & & Ajustada para sexo e IMC & \\
\hline & & & PAS & 0,38 \\
\hline & & & PAD & $>0,05$ \\
\hline
\end{tabular}

(continua) 
Tabela 2 (continuação)

\begin{tabular}{|c|c|c|c|c|}
\hline Artigo & Desfecho & Variáveis de interesse & Análise estatística & Valor de $p$ \\
\hline \multirow[t]{26}{*}{ Willemsen et al. 32} & Hipertensão arterial & Prematuridade & Regressão linear não & \\
\hline & & & ajustada & \\
\hline & & & PAS & 0,010 \\
\hline & & & PAD & $<0,0005$ \\
\hline & & & Regressão logística, modelo & \\
\hline & & & comprimento ao nascer & \\
\hline & & & PAS & 0,003 \\
\hline & & & PAD & 0,058 \\
\hline & & & Regressão logística, modelo & \\
\hline & & & 2 ajustado por idade, sexo, & \\
\hline & & & PAS & 0,003 \\
\hline & & & PAD & 0,026 \\
\hline & & & Coeficiente de correlação de & \\
\hline & & & Spearman & \\
\hline & & & $\mathrm{R}^{2}$ & \\
\hline & & & PAS & 0,08 \\
\hline & & & PAD & 0,05 \\
\hline & & & $\mathrm{R}^{2}$ ajustado & \\
\hline & & & PAS & 0,07 \\
\hline & & & PAD & 0,05 \\
\hline & & & Modelo 2 & \\
\hline & & & PAS > p95 & 0,022 \\
\hline & & & PAD > p95 & 0,713 \\
\hline & & & Regressão linear múltipla & \\
\hline & & & PAS & 0,001 \\
\hline & & & PAD & 0,023 \\
\hline
\end{tabular}

DCV: doença cardiovascular; IMC: índice de massa corporal; PA: pressão arterial; PAD: pressão arterial diastólica; PAS: pressão arterial sistólica; PIG: pequeno para a idade gestacional; VOP: velocidade de onda de pulso.

Outro fato observado foram as diferenças metodológicas utilizadas entre os estudos, o que dificultou a comparação entre os mesmos, diferenças estas, referentes à população estudada, ao método de aferição da pressão arterial, ao parâmetro utilizado para determinar a prematuridade e às análises estatísticas realizadas.

Dos 9 artigos analisados, em apenas 2, ambos transversais, foi possível observar associa- ção estatisticamente significante entre prematuridade e aumento da pressão arterial. Um desses estudos foi realizado com crianças e adolescentes, tendo sido encontrada associação estatisticamente significante somente entre aumento da PAS e prematuridade 16; no outro estudo, realizado somente com crianças, foi encontrada associação significativa entre aumento da pressão arterial e prematuridade apenas em crianças 
Fatores de confundimento estudados nos artigos selecionados.

\begin{tabular}{|c|c|c|c|c|c|c|c|c|c|}
\hline $\begin{array}{l}\text { Fatores de } \\
\text { confundimento }\end{array}$ & $\begin{array}{l}\text { Cheung } \\
\text { et al. } 8\end{array}$ & $\begin{array}{c}\text { Johansson } \\
\text { et al. } 12\end{array}$ & $\begin{array}{l}\text { Relton } \\
\text { et al. } 16\end{array}$ & $\begin{array}{c}\text { Bracewell } \\
\text { et al. } 31\end{array}$ & $\begin{array}{l}\text { Woelk } \\
\text { et al. } 33\end{array}$ & $\begin{array}{c}\text { Willemsen } \\
\text { et al. } 32\end{array}$ & $\begin{array}{l}\text { Bonamy } \\
\text { et al. } 26\end{array}$ & $\begin{array}{c}\text { Bayrakci } \\
\text { et al. } 9\end{array}$ & $\begin{array}{l}\text { Rondó } \\
\text { et al. } 30\end{array}$ \\
\hline Idade & & & $x$ & & & & $x$ & & \\
\hline Altura atual & & $x$ & & $x$ & & $x$ & $x$ & & \\
\hline Idade atual & $x$ & & & & & & & & \\
\hline Peso ao nascer & & & $x$ & & & $x$ & & & \\
\hline IMC & & & $x$ & $x$ & & $x$ & & & \\
\hline VOP & $x$ & & & & & & & & \\
\hline Eclâmpsia & & $x$ & & & & & & & \\
\hline Peso atual & & & & & $x$ & & & & \\
\hline Sexo & & & & & & $x$ & $x$ & & \\
\hline Etnia & & & & & & $x$ & & & \\
\hline Comprimento ao & & & & & & $x$ & & & \\
\hline \multicolumn{10}{|l|}{ nascer } \\
\hline História familiar & & & & & & & $x$ & & \\
\hline para DCV & & & & & & & & & \\
\hline
\end{tabular}

DCV: doença cardiovascular; IMC: índice de massa corporal; VOP: velocidade de onda de pulso.

prematuras PIG, mesmo após o ajuste pelo peso e comprimento ao nascer, IMC e estatura 28. Apesar do estudo de Relton et al. 16 ter sido feito com desenho transversal, no qual a exposição e desfecho são avaliados simultaneamente, não havendo portanto o acompanhamento dos indivíduos ao longo do tempo, foi utilizado um método mais fidedigno de mensuração dos níveis pressóricos - a MAPA -, o que propicia uma visão dinâmica do comportamento tensional. Outro fator relevante foi a utilização, em ambos os estudos, da ultrassonografia e do cálculo da data da última menstruação, fornecidos pelos pais das crianças, para a avaliação da idade gestacional, o que proporciona uma melhor estimativa desse parâmetro. Além desse fato, esses estudos foram os que melhor analisaram as interferências de importantes fatores de confundimento na associação entre pressão arterial e prematuridade, como a idade atual, o peso ao nascer, o IMC, o comprimento ao nascer e o sexo.

Os estudos de coorte e caso-controle não detectaram associação significativa entre pressão arterial e prematuridade. É necessário, porém, mencionar que as amostras utilizadas nos estudos de caso-controle foram pequenas 8,9,34 e no estudo de coorte prospectiva o número de prematuros encontrados foi ainda menor, apenas 27 crianças 33 , fato este que pode interferir no julgamento dos resultados obtidos.

$\mathrm{O}$ fato de estudos realizados com adultos demonstrarem maior relação entre hipertensão arterial e prematuridade do que em estudos com crianças sugere que os efeitos da prematuridade poderiam incidir em fatores indiretos, os quais irão contribuir para a elevação da pressão arterial somente na fase adulta, tais como a disfunção arterial e a hiperatividade do sistema simpáticoadrenal. Relton et al. 16 reforçam essa idéia ao observar a relação entre redução da idade gestacional com elevada pressão de pulso (estimada pela diferença entre as médias das mensurações das PAS e PAD de 24 horas) em crianças do sexo feminino, sugerindo que o aumento da rigidez arterial pode estar relacionado ao aparecimento da hipertensão arterial no adulto. É necessário, porém, avaliar os achados com cautela devido à escassez de estudos que testaram essa hipótese.

O peso ao nascer foi analisado em associação com a prematuridade na elevação da pressão arterial em 3 dos 9 estudos, dos quais 2 estudos encontraram associação estatisticamente significativa entre peso ao nascer e pressão arterial, independentemente da idade gestacional. Cheung et al. 8 encontraram valores mais altos de pressão arterial somente no grupo de prematuros PIG. Além disso, mostrou que rigidez arterial periférica está aumentada em crianças prematuras e PIG. Em concordância com o estudo anterior, Willemsen et al. 32 observaram que prematuros e PIG apresentaram valores significativamente maiores de PAS e PAD do que crianças nascidas a termo e PIG. Elevação na freqüência cardíaca em crianças prematuras e PIG quando 
comparadas com os controles foi observada por Johansson et al. 21. Outros estudos com crianças também demonstram essa associação, como os de Fattal-Valesvski et al. 27, Pereira et al. 35 e Salgado et al. 36 .

O fato de não ter sido observado que a prematuridade seja um fator preditor para elevação da pressão arterial na infância na maioria dos estudos desta revisão, não descarta a hipótese de que haja alguma interferência deste fator nos níveis pressóricos na infância, visto que os estudos que encontraram associação foram os que utilizaram métodos mais fidedignos de mensuração da idade gestacional (ultrassonografia) e de medida da pressão arterial (um deles utilizou a MAPA) e controlaram suas análises por fatores importantes de confundimento. Nos estudos que não encontram associação, apesar de serem do tipo coorte ou caso-controle, a maioria deles utilizou amostras muito pequenas, o que poderia ter diminuído o poder do estudo, além disto, ajustaram seus re-

\section{Resumo}

Estudos epidemiológicos têm sugerido que a hipertensão arterial é uma doença que tem início na infância e a prematuridade, crianças nascidas com menos de 37 semanas, um possível fator associado ao desenvolvimento deste agravo. Este estudo tem por objetivo identificar a associação entre prematuridade e elevação da pressão arterial em crianças por meio de revisão sistemática da literatura. Foram selecionados artigos das bases de dados MEDLINE, LILACS e SciELO publicados no período de 1998 a 2009. Excluiu-se os que não apresentavam resumo, redigidos em idioma diferente do inglês, português e espanhol e os que não objetivavam analisar a relação entre prematuridade e hipertensão. Foram analisados 9 artigos: 5 estudos de caso-controle, 2 transversais e 2 de coorte. Prematuridade não esteve associada à elevação da pressão arterial na infância na maioria dos artigos analisados, porém, sua influência não deve ser descartada, tendo em vista a pequena produção sobre o assunto, bem como as grandes diferenças metodológicas observadas nos estudos publicados no período analisado.

Hipertensão; Prematuro; Criança sultados por poucos ou nenhum fator relevante de confundimento, como peso ao nascer, comprimento ao nascer, peso e altura atuais.

É necessário, contudo, a realização de mais pesquisas sobre o tema, principalmente com amostras maiores, tendo em vista que em adultos essa associação já está bem estabelecida. Portanto, o resultado esperado de encontrar associação entre o aumento da pressão arterial em crianças prematuras acaba sendo questão de tempo ou de poder de estudo. Na infância, a diferença na pressão arterial entre crianças prematuras e aquelas nascidas a termo seria pequena e esta tenderia a se ampliar na medida em que a vida adulta fosse chegando. Logo, para se detectar pequena diferença na pressão arterial na infância, há necessidade de estudos com grandes amostras e métodos mais precisos de medida, o que torna mais complexo o estudo da associação entre prematuridade e hipertensão arterial em crianças.

\section{Colaboradores}

A. P. Coelli e L. R. Nascimento contribuíram na concepção, análise dos dados e redação do manuscrito. J. G. Mill contribuiu na revisão crítica do manuscrito. M. C. B. Molina contribuiu na concepção e na revisão crítica do manuscrito. 


\section{Referências}

1. Bao W, Srinivisan SR, Wattigney WA, Berenson GS The relation of parental cardiovascular disease to risk factores in children and young adults: The Bogalusa Heart Study. Circulation 1995; 91:365-71.

2. Sorof JM, Lai D, Turner J, Poffenvarger T, Portman RJ. Overweight, ethnicity, and the prevalence of in school aged children. Pediatrics 2004; 113:475-82.

3. Rezende DF, Scarpelli RAB, Souza GF, Costa JO, Scarpelli MB, Scarpelli PA, et al. Prevalência da hipertensão arterial sistêmica em escolares de 7 a 14 anos do Município de Barbacena, Minas Gerais, em 1999. Arq Bras Cardiol 2003; 81:375-80.

4. Sorof JM, Daniels S. Obesity hypertension in children: a problem of epidemic proportions. Hypertension 2002; 40:441-7.

5. Salgado CM, Carvalhães JTA. Hipertensão arterial na infância. J Pediatr (Rio J.) 2003; 79 Suppl 1: S115-23.

6. Dim-Dzietham R, Liu Y, Bielo MV, Samsa F. High blood pressure trends in children and adolescents in national surveys, 1963 to 2002. Circulation 2007; 116:1488-96.

7. Global Alliance to Prevent Prematurity and Stillbirth Resources. Facts \& figures. http://www. gapps.org/resources/\#prematurity (acessado em 18/Jun/2009)

8. Cheung YF, Wong KY, Lam BC, Tsoi NS. Relation of arterial stiffness with gestational age and birth weight. Arch Dis Child 2004; 89:217-21.

9. Bayrakci US, Schaefer F, Duzova A, Yigit S, Bakkaloglu A. Abnormal circadian blood pressure regulation in children born preterm. J Pediatr 2007; 151:399-403.

10. Rakow A, Johansson S, Legnevall L, Sevastik R, Celsi G, Norman M, Vanpee M. Renal volume and function in school-age children born preterm or small for gestational age. Pediatr Nephrol 2008; 23:1309-15.

11. Irving RJ, Belton NR, Elton RA, Walker BR. Adult cardiovascular risk factors premature babies. Lancet 2000; 355:2135-6.

12. Johansson S, Iliadou A, Bergvall N, Tuvemo T, Norman M, Cnattingius S. Risk of high blood pressure among young men increases with the degree of immaturity at birth. Circulation 2005: 112:3430-6.

13. Dalziel SR, Parag V, Rodgers A, Harding JE. Cardiovascular risk factors at age 30 following pre-term birth. Int J Epidemiol 2007; 36:907-15.

14. Beck S, Wojdyla LS, Betran AP, Merialdi M, Requejo $\mathrm{JH}$, Rubens C, et al. The worldwide incidence of preterm birth: a systematic review of maternal mortality and morbidity. Bull World Health Organ 2010; 88:31-8

15. Ministério da Saúde. Informações e análises. Uma análise dos nascimentos no Brasil e regiões. http://portal.saude.gov.br/portal/saude/Gestor/ visualizar_texto.cfm?idtxt=24455 (acessado em 16/Jun/09)

16. Relton CL, Pearce MS, O'Sullivan JJ. The relationship between gestational age, systolic blood pressure and pulse pressure in children. J Hum Hypertens 2008; 22:352-7.
17. Lawor DA, Hubinette A, Tynelius O, Leon DA, Smith GD, Rasmussem F. Associations of gestational age and intrauterine growth with systolic blood pressure in a family-based study of 386485 men in 331089 families. Circulation 2007; 115:562-8.

18. Kistner A, Jacobson L, Jacobson SH, Svensson E, Hellstron A. Low gestational age associated with abnormal retinal vascularization and increased blood pressure in adult women. Pediatr Res 2002; 51:675-80.

19. Gournay V, Droin E, Rozé JC. Development of baroreflex control of heart rate in preterm and full term infants. Arch Dis Child Fetal Neonatal Ed 2002; 86:F151-4.

20. Kistner A, Celsi G, Vanpee M, Jacobson SH. Increased blood pressure but normal renal function in adult women born preterm. Pediatr Nephrol $2000 ; 15: 215-20$.

21. Johansson S, Norman M, Legnevall L, Dalmaz $H$, Lagercrantz $H$, Vampe $M$. Increased catecholamines and heart rate in children with low birth weight: perinatal contributions to sympathoadrenal overactivity. J Intern Med 2007; 261:480-7.

22. Lurbe E, Redón I, Tacons J, Torro I, Alvarez V. Current and birth weights exert independent influences on nocturnal pressure-natriuresis relationships in normotensive children. Hypertension 1998; 31:546-51.

23. Veening MA, Weissenbruch MMV, Waal HAD. Sequelae of sydrome $\mathrm{X}$ in children born small for gestacional age. Horm Res 2004; 61:103-7.

24. Barker DJP, Bull AR, Osmond C, Simmond SJ. Fetal and placental size and risk of hypertension in adult life. BMJ 1990; 301:259-62.

25. Lei $\mathrm{n}^{\circ}$. 8.069, de 13 de julho de 1990. Estatuto da Criança e do Adolescente. http://www.planalto. gov.br/ccivil/LEIS/L8069.htm (acessado em 16/ Set/2009).

26. Bonamy AK, Andolf E, Martin H, Norman M. Preterm birth and carotid diameter and stiffness in childhood. Acta Paediatr 2008; 97:434-7.

27. Fattal-Valevski A, Bernheim J, Leitner Y, Redianu B, Bassan H, Harel S. Blood pressure values in children with intrauterine growth retardation. Isr Med Assoc J 2001; 3:805-8.

28. De Rogalski LI, Roche F, Pichot V, Teyssier G, Gaspoz JM, Barthelemy JC. Autonomic nervous system activity in premature and full-term infants from theoretical term to 7 years. Auton Neurosci 2007; 136:105-9.

29. Bergel E, Haelterman E, Belizán J, Villar J, Carroli G. Perinatal factors associated with blood pressure during childhood. Am J Epidemiol 2000; 151:594601.

30. Rondó PH, Lemos JO, Pereira JA, Oliveira JM, Innocente LR. Relationship between birthweight and arterial elasticity in childhood. Clin Sci (Lond) 2008; 115:317-26.

31. Bracewell MA, Hennessy EM, Wolke D, Marlow N. The EPICure study: growth and blood pressure at 6 years of age following extremely preterm birth. Arch Dis Child Fetal Neonatal Ed 2008; 96:108-14. 
32. Willemsen RH, de Kort SW, van der Kaay DC, Hokken-Koelega AC. Independent effects of prematurity on metabolic and cardiovascular risk factors in short small-for-gestational-age children. J Clin Endocrinol Metab 2008; 93:452-8.

33. Woelk G, Emanuel I, Weiss NS, Psaty BM. Birthweight and blood pressure among children in Harare, Zimbabwe. Arch Dis Child Fetal Neonatal Ed 1998; 79:119-22.

34. Bonamy AK, Martin H, Jorneskog G, Norman M. Lower skin capillary density, normal endothelial function and higher blood pressure in children born preterm. J Intern Med 2007; 262:635-42.
35. Pereira C. Baixo peso ao nascimento e hipertensão arterial na infância: estudo epidemiológico de base comunitária. Millenium 2004; 30:59-73.

36. Salgado MC, Jardim PCBV, Teles FBG, Nunes MC. Baixo peso ao nascer como marcador de alterações na Monitorização Ambulatorial da Pressão Arterial. Arq Bras Cardiol 2009; 92:113-21.

Recebido em 17/Mar/2010

Versão final reapresentada em 14/Out/2010 Aprovado em 14/Jan/2011 